\title{
Increased Cellular Aging by 3 Years of Age in Latino, Preschool Children Who Consume More Sugar-Sweetened Beverages: A Pilot Study
}

\author{
Janet M. Wojcicki, PhD, MPH, MAS,' Rosalinda Medrano, BA, \\ Jue Lin, $\mathrm{PhD}^{2}$, and Elissa Epel, $\mathrm{PhD}^{3}$
}

\section{Abstract}

Background: Previous studies in adults and older children find that sugar-sweetened beverage (SSB) consumption increases risk for obesity and cellular aging, as measured by leukocyte telomere length (LTL).

Methods: In a previously described, San Francisco-based Latino birth cohort, where telomere length was measured at birth, we evaluate the relationship between beverage consumption (including SSB and $100 \%$ fruit juice), obesity, and LTL at 2-3 years old, as well as change in LTL from birth. LTL (T/S Ratio) was measured in 61 children (mean 2.4 years \pm 0.6 standard deviation). Multivariable linear regression models are used to ascertain beverage type and obesity as independent predictors of LTL and change in LTL.

Results: Mean telomere length was $1.58 \pm 0.20$ (T/S Ratio) and mean yearly change was $-0.08 \pm-0.09$ (T/S Ratio). Predictors of shorter telomere length at age 2-3 included increased consumption of SSB (Beta Coeff $=-0.009$ 95\% CI [ -0.02 to -0.0008$]$; $p=0.03$ ). Telomere length at birth was the strongest predictor of rate of attrition from birth to $2-3$ years of age and males tended to have more rapid attrition.

Conclusion: Excessive SSB consumption impacts early childhood immune system health adversely, possibly before the development of obesity.

Keywords: obesity; soda; SSB; telomere

\section{Background}

\section{Sugar-Sweetened Beverages and Obesity}

A number of previous studies have demonstrated an association between sugar-sweetened beverages (SSB) consumption and adverse outcomes in children, particularly obesity and unhealthy weight gain. ${ }^{1,2}$ Public health interventions have sought to delay the introduction of SSB as well as amount consumed as means to address the obesity epidemic. ${ }^{3}$ However, there appears to be an age effect with SSB consumption, as some studies do not show an association between SSB and obesity in younger preschool children even in the context of high consumption ( $\geq 1$ serving/day). ${ }^{4}$ It is possible a number of years of consumption are needed before development of obesity or metabolic changes precede the development of obesity. In our study, we sought to investigate the role of SSB consumption in the first 2-3 years of life on leukocyte telomere length (LTL) at 2 years, as a possible marker of the adverse impact of SSBs on metabolic health, in relation to child obesity.

\section{LTL as Biomarker of Inflammation}

Telomeres are the repetitive nucleotide sequences that form the caps on chromosomes, helping prevent degradation and abnormal recombination of DNA strands. With each cell division, telomeres shorten, but the process is accelerated through exposure to oxidative stress and inflammation. Shortened telomeres may accelerate aging through increased production of cytokines, starting an inflammatory cascade. Shorter telomere length has been shown to be associated with a number of adverse cardiometabolic outcomes, such as stroke, myocardial infarction, and type 2 diabetes mellitus. ${ }^{5}$

\section{SSB's and Telomere Length}

Previous studies have investigated the relationship between shortened telomere length and SSB consumption, 
primarily in adults. Findings in three studies have been mixed with three positive findings and one null finding. One study found that sugar-sweetened carbonated soda consumption was associated with increased risk for shorter telomere length in adults using National Health and Nutrition Examination Study data (NHANES) $(n=5309){ }^{6}$ whereas a smaller multiethnic cohort $(n=840)$ did not find an association between sugar-sweetened soda intake and telomere length. ${ }^{7}$ Additionally, a study of 1958 Korean middle-aged adults found that greater SSB consumption were associated with shorter telomere length, when measured 10 years later. ${ }^{8}$ Additionally, there have been two secondary analyses that uncovered relations between SSBs and LTL over time. Our previous study with another San Francisco-based Latino cohort of children found that high levels of sugar-sweetened soda consumption at 3 years of age was associated with accelerated attrition from 4 to 5 years of age ${ }^{9}$ and in a pregnant cohort reductions in SSB intake were associated with apparent telomere lengthening. ${ }^{10}$

As the childhood years, particularly those from 0 to 4 years, are associated with the greatest telomere length attrition compared with later childhood and adulthood, ${ }^{11-13}$ our study sought to investigate the relationship between telomere length at 2-3 years, telomere length attrition from 0 to 2-3 years, and SSB consumption from birth. These early years of childhood are the time when sodas and other sugared beverages are first being introduced and beverage consumption patterns are being formed, and as such may provide an indication of the role of these beverages on cellular aging in young children in different weight categories.

\section{Methods}

The Latino, Eating and Diabetes cohort (LEAD) has been previously described in other publications, including specifics of inclusion, exclusion, and recruitment. ${ }^{14,15}$ Briefly, mothers were recruited during pregnancy at Zuckerberg San Francisco General Hospital (ZSFGH) and followed up through delivery until 3 years of age. Mothers who had any contraindication for breastfeeding or reported drug or alcohol abuse in pregnancy were excluded from the study. Follow-up was conducted at birth, 4-6 weeks, 6 months, 12 months, and annually thereafter until age 3 . Cord blood was collected at birth so as to measure LTL and dietary intake and weight and length/height and waist circumference parameters were collected at 4-6 weeks of age, 6 months, 12 months, and annually thereafter. Whole blood for LTL analysis was subsequently collected through venipuncture blood draw between 2 and 3 years of age. All mothers provided written consent for themselves and their children. The study was approved by the Committee on Human Research (CHR), the Institutional Review Board of the University of California, San Francisco.

\section{Telomere Length Assays}

Genomic DNA was purified from cord blood collected at birth and venous blood collected at 2-3 years with the QIAamp DNA Blood Mini Kit (Cat\# 51106; Qiagen, Hilden,
Germany). Acceptance criteria were OD260/OD280 between 1.7 and 2.0 and concentration greater than $20 \mathrm{ng} / \mu \mathrm{L}$. Telomere length is expressed as $\mathrm{T} / \mathrm{S}$ (the ratio of telomeric product vs. single-copy gene product). The quantitative polymerase chain reaction (qPCR) telomere length measurement assay was adapted from the published original method by Cawthon ${ }^{16}$ and Lin et al. ${ }^{17}$ and as previously described in Wojcicki et al., 9,11 To control for assay batch difference between the cord blood samples and year 2-3 samples, DNA was extracted again from 32 cord blood samples separately stored at $-80^{\circ} \mathrm{C}$ and assayed together with the year 2-3 samples. An adjustment factor was derived by comparing the current cord blood sample $\mathrm{T} / \mathrm{S}$ ratios to those initially assayed and applied to the current year $2-3$ samples. ${ }^{14}$

\section{Outcome}

Our primary outcome of interest was telomere length (T/S Ratio) at 2-3 years of age. Our secondary outcome of interest included telomere length change from birth to 2-3 years of age adjusted for years of age at the second time point of collection.

\section{Predictors}

Our primary predictor of interest was SSB consumption (defined as soda, Kool-Aid, Hi-C, sweetened juices, and other beverages with added sugar), including age of introduction and servings consumed, and secondary predictors included other beverages, such as $100 \%$ fruit juice, flavored milks, and duration of breastfeeding and exclusive breastfeeding. At 4-6 weeks, 6 months, and 12 months we collected information on introduction of beverages only, not quantity consumed. At 24 months of age, we collected information on frequency of consumption per month. We analyzed beverage consumption as age/timing of introduction of beverages as well as frequency of consumption at 24 months of age, including SSB, flavored milks, and $100 \%$ fruit juice analyzed continuously and dichotomously ( $\geq 3 \times /$ week and less). We did not collect data on total quantity consumed, but rather parents responded how frequently children consumed specific items.

Secondary predictors of interest included child weight and obesity at different time points $(6,12$, and 24-36 months) defining obesity as $\geq 95$ th percentile using weight for length measurements (for children under 2 years) and body mass index (BMI) (for children 2 years and older) using the Centers of Disease Control (CDC) growth curves. ${ }^{18} \mathrm{We}$ also assessed birthweight in grams and weight for length $\mathrm{Z}$ score at birth ${ }^{18}$ in relation to telomere length at 2-3 years of age. We additionally assessed abdominal obesity defined as $\geq 90$ th percentile in relation to LTL. ${ }^{19}$

\section{Covariates}

Primary covariates that we assessed in relation to our outcomes of interest included maternal education level (high school diploma or less education than a high school diploma), maternal age (continuous and advanced $[\geq 35$ years and $<35]$ ), child sex (male vs. female), total household income ( $\geq \$ 25,000 /$ year versus $<\$ 25,000 /$ year), and maternal 
ethnicity (Mexican vs. Central American origin based on self-report) as previously reported. ${ }^{19}$

\section{Statistical Analysis}

As our outcome of interest was LTL at 2-3 years of age and change in LTL from birth to $2-3$, we graphically assessed the distribution of the data for both outcomes. Both outcomes were normally distributed as indicated by the Shapiro-Wilk, Shapiro-Francia, and the Skewness-Kurtosis tests. We used $t$-tests to assess relationships between dichotomous predictors and mean LTL at age 2-3. As our outcome was smaller for change in LTL from age 0 to $2-3$ years, we used a nonparametric assessment, Wilcoxon two-sample sign rank test. Linear regression was used for continuous predictors. Variables that were significant at $p<0.10$ in bivariate analysis as were those that we were particularly interested to assess (SSB) were included in multivariable models with the exception of variables that had fewer than five observations in cells. We subsequently evaluated any potential confounding and interactive relationship between obesity and abdominal obesity status and SSB consumption using interaction terms in our models given previous findings that SSB consumption and obesity are associated with accelerated telomere attrition, ${ }^{9,10}$ and the association between SSB consumption and weight status in children. ${ }^{4}$ Proportional distribution of select variables of interest, including SSB consumption were plotted using $\mathrm{K}$ density curves in Stata 13.0.

\section{Results}

\section{Predictors of Telomere Length at 2-3 Years}

There were 61 children who had LTL measured at 2-3 years of age (mean 2.4 years \pm 0.6 standard deviation [SD]). Mean telomere length was $0.58 \pm 0.20 \mathrm{~T} / \mathrm{S}$ Ratio (Table 1).

Sociodemographic factors. We did not see any differences in telomere length at 2-3 years, based and demographic factors, including maternal education level, maternal

Table I. Child Leukocyte Telomere Length at 2-3 Years $(N=6 I)$ and Change from 0 to 2-3 Years $(n=30)$ in Relation to Sociodemographics and Perinatal Risk Factors

\begin{tabular}{|c|c|c|c|c|c|c|}
\hline Variable & $\begin{array}{c}\text { Variable } \\
\text { mean } \pm \text { SD } \\
\text { or } \mathrm{N} / \mathrm{T}(\%)\end{array}$ & $\begin{array}{l}\text { Telomere length } \\
\text { mean } \pm \text { SD } \\
\text { (T/S ratio) }\end{array}$ & $p$ & $\begin{array}{c}\text { Variable } \\
\text { mean } \pm \text { SD } \\
\text { or }(\%)\end{array}$ & $\begin{array}{c}\text { Telomere } \\
\text { length change } \\
\text { median } \pm \text { SD } \\
\text { (yearly) (T/S ratio) }\end{array}$ & $\mathbf{p}$ \\
\hline Telomere length & & $1.58 \pm 0.20$ & & & & \\
\hline Telomere length change & & & & & $-0.08 \pm 0.09$ & \\
\hline \multicolumn{7}{|l|}{ Child and family sociodemographics } \\
\hline \multicolumn{7}{|l|}{ Child sex } \\
\hline Female & $31 / 6 I(5 I .7)$ & $1.61 \pm 0.18$ & & $14 / 30(46.7)$ & $-0.03 \pm 0.10$ & \\
\hline Male & $30 / 61$ (49.2) & $1.55 \pm 0.21$ & 0.20 & $16 / 30(53.3)$ & $-0.09 \pm 0.09$ & 0.21 \\
\hline \multicolumn{7}{|l|}{ Maternal education } \\
\hline Less than high school & $43 / 59$ (72.9) & $1.57 \pm 0.21$ & & $18 / 30(60.0)$ & $-0.06 \pm 0.09$ & \\
\hline High school graduate or more & $16 / 59(27.1)$ & $1.60 \pm 0.18$ & 0.62 & $12 / 30(40.0)$ & $-0.15 \pm 0.09$ & 0.06 \\
\hline \multicolumn{7}{|l|}{ Maternal ethnicity } \\
\hline Mexican & $32 / 61$ (52.5) & $1.57 \pm 0.18$ & & $17 / 30(56.7)$ & $-0.08 \pm 0.11$ & \\
\hline Central American & 29/6I (47.5) & $1.59 \pm 0.22$ & 0.73 & $13 / 30(43.3)$ & $-0.10 \pm 0.08$ & 0.95 \\
\hline Maternal age & $28.0 \pm 5.3$ & & & & & \\
\hline$<35$ years & $5 I / 6 I(85.0)$ & $1.58 \pm 0.20$ & & $26 / 30(86.7)$ & $-0.07 \pm 0.08$ & \\
\hline$\geq 35$ years & $10 / 61(16.4)$ & $1.58 \pm 0.17$ & 0.98 & $4 / 30(13.3)$ & $-0.14 \pm 0.16$ & 0.83 \\
\hline \multicolumn{7}{|l|}{ Marital status } \\
\hline Single or divorced/separated/widowed & $9 / 61(14.8)$ & $1.57 \pm 0.17$ & & $2 / 30(6.7)$ & $-0.02 \pm 0.15$ & \\
\hline Single, living with partner or married & $52 / 61$ (85.2) & $1.58 \pm 0.20$ & 0.82 & $28 / 30(93.3)$ & $-0.08 \pm 0.09$ & 0.45 \\
\hline \multicolumn{7}{|l|}{ Household income } \\
\hline$<\$ 25,000 /$ year & $55 / 61(90.0)$ & $1.58 \pm 0.20$ & 0.73 & $27 / 30(90.0)$ & $-0.07 \pm-0.09$ & 0.18 \\
\hline$\geq \$ 25,000 /$ year & $6 / 61(9.8)$ & $1.55 \pm 0.22$ & & $3 / 30(10.0)$ & $-0.14 \pm 0.07$ & \\
\hline
\end{tabular}

$\mathrm{SD}$, standard deviation. 
Table 2. Child Leukocyte Telomere Length at 2-3 Years $(n=6 \mathrm{I})$ and Change from 0 to 2-3

Years $(n=30)$ in Relation to Weight Characteristics and Beverage Intake

\begin{tabular}{|c|c|c|c|c|c|c|}
\hline Variable & N/T (\%) & \begin{tabular}{|l|} 
Leukocyte \\
telomere length, \\
mean $\pm \mathrm{SD}$ \\
(T/S ratio)
\end{tabular} & $\mathbf{p}$ & $\begin{array}{l}\text { Mean } \pm \text { SD } \\
\text { or }(\%)\end{array}$ & $\begin{array}{l}\text { Leukocyte telomere } \\
\text { length change } \\
0-2 \text { years, } \\
\text { median } \pm \text { SD } \\
\text { (T/S ratio) }\end{array}$ & $p$ \\
\hline \multicolumn{7}{|c|}{ Child weight characteristics } \\
\hline \multicolumn{7}{|c|}{ Obese at 6 months } \\
\hline Yes & $8 / 61(13.1)$ & $1.60 \pm 0.20$ & & $6 / 30(20.0)$ & $-0.07 \pm 0.11$ & \\
\hline No & $53 / 61(86.9)$ & $1.46 \pm 0.14$ & 0.06 & $24 / 30(80.0)$ & $-0.08 \pm 0.09$ & 0.53 \\
\hline \multicolumn{7}{|c|}{ Obese at 12 months } \\
\hline Yes & $9 / 61(14.8)$ & $1.63 \pm 0.25$ & & $4 / 30(13.3)$ & $-0.05 \pm 0.14$ & \\
\hline No & $52 / 61(85.2)$ & $1.57 \pm 0.40$ & 0.40 & $26 / 30(86.7)$ & $-0.08 \pm 0.09$ & 0.67 \\
\hline \multicolumn{7}{|c|}{ Obese at $2-3$ years } \\
\hline Yes & $7 / 59(11.9)$ & $1.53 \pm 0.17$ & & $3 / 30(10.0)$ & $-0.03 \pm 0.06$ & \\
\hline No & $52 / 59(88.1)$ & $1.59 \pm 0.20$ & 0.46 & $27 / 30(90.0)$ & $-0.10 \pm 0.06$ & 0.14 \\
\hline \multicolumn{7}{|c|}{ Obese at any time from 6 months to $2-3$ years } \\
\hline Yes & $23 / 59(39.0)$ & $1.60 \pm 0.22$ & 0.61 & $10 / 30(33.3)$ & $-0.07 \pm 0.11$ & \\
\hline No & $36 / 59(61.0)$ & $1.57 \pm 0.19$ & & $20 / 30(66.7)$ & $-0.08 \pm 0.09$ & 0.54 \\
\hline \multicolumn{7}{|c|}{ High waist at $2-3$ years ( $>90$ th) } \\
\hline Yes & $17 / 58(29.3)$ & $1.55 \pm 0.05$ & & $9 / 28(32.1)$ & $-0.13 \pm 0.09$ & \\
\hline No & $4 \mathrm{I} / 58(70.6)$ & $1.58 \pm 0.19$ & 0.66 & 19/28 (67.9) & $-0.08 \pm 0.09$ & 0.48 \\
\hline \multicolumn{7}{|c|}{ Dietary intake } \\
\hline \multicolumn{7}{|c|}{ Exclusive breastfeeding at 4-6 weeks } \\
\hline Yes & $22 / 61(36.1)$ & $1.56 \pm 0.18$ & & $12 / 30(40.0)$ & $-0.04 \pm 0.09$ & \\
\hline No & $39 / 61(63.9)$ & $1.59 \pm 0.21$ & 0.56 & $18 / 30(60.0)$ & $-0.11 \pm 0.10$ & 0.40 \\
\hline \multicolumn{7}{|c|}{ Other nonmilk beverage consumption at 4-6 weeks } \\
\hline Yes & $|4 / 6|(23.0)$ & $1.59 \pm 0.23$ & & $6 / 30(20.0)$ & $-0.11 \pm 0.07$ & \\
\hline No & $47 / 61(77.0)$ & $1.58 \pm 0.19$ & 0.85 & $24 / 30(80.0)$ & $-0.07 \pm 0.10$ & 0.84 \\
\hline \multicolumn{7}{|c|}{ Any breastfeeding at 6 months } \\
\hline Yes & $43 / 58(74.1)$ & $1.59 \pm 0.21$ & & $23 / 39(59.0)$ & $-0.08 \pm 0.09$ & \\
\hline No & $15 / 58(25.9)$ & $1.53 \pm 0.17$ & 0.33 & $6 / 29(20.7)$ & $-0.06 \pm 0.11$ & 0.52 \\
\hline \multicolumn{7}{|c|}{ Any breastfeeding at 12 months } \\
\hline Yes & $38 / 60(63.3)$ & $1.60 \pm 0.21$ & & $20 / 29(69.0)$ & $-0.06 \pm 0.09$ & \\
\hline No & $22 / 60(36.7)$ & $1.55 \pm 0.17$ & 0.31 & $9 / 29(31.0)$ & $-0.10 \pm 0.11$ & 0.48 \\
\hline \multicolumn{7}{|c|}{ Any $100 \%$ fruit juice 6 months } \\
\hline Yes & 16/57 (28.1) & $1.55 \pm 0.24$ & & $8 / 28(28.6)$ & $-0.13 \pm 0.06$ & \\
\hline No & $41 / 57(71.9)$ & $1.59 \pm 0.18$ & 0.58 & $20 / 28(71.4)$ & $-0.05 \pm 0.10$ & 0.17 \\
\hline \multicolumn{7}{|c|}{ Daily $100 \%$ fruit juice at 2 years } \\
\hline Yes & 19/57 (29.8) & $1.55 \pm 0.21$ & & $9 / 29(31.0)$ & $-0.13 \pm 0.10$ & \\
\hline \multirow[t]{2}{*}{ No } & $38 / 57(67.8)$ & $1.58 \pm 0.17$ & 0.62 & $20 / 29(69.0)$ & $-0.07 \pm 0.09$ & 0.92 \\
\hline & \multicolumn{6}{|c|}{ continued on page 153} \\
\hline
\end{tabular}


Table 2. Child Leukocyte Telomere Length at 2-3 Years $(n=6 \mathrm{I})$ and Change from 0 to 2-3

Years $(n=30)$ in Relation to Weight Characteristics and Beverage Intake continued

\begin{tabular}{|c|c|c|c|c|c|c|}
\hline Variable & N/T (\%) & $\begin{array}{l}\text { Leukocyte } \\
\text { telomere length, } \\
\text { mean } \pm \mathrm{SD} \\
\text { (T/S ratio) }\end{array}$ & $p$ & $\begin{array}{l}\text { Mean } \pm \text { SD } \\
\text { or }(\%)\end{array}$ & $\begin{array}{c}\text { Leukocyte telomere } \\
\text { length change } \\
0-2 \text { years, } \\
\text { median } \pm \text { SD } \\
\text { (T/S ratio) }\end{array}$ & $p$ \\
\hline \multicolumn{7}{|c|}{ Any $\mathrm{SSBs}^{\mathrm{a}}$ at 12 months } \\
\hline Yes & $15 / 60(25.0)$ & $1.54 \pm 0.19$ & & $7 / 29(24.1)$ & $-0.07 \pm 0.11$ & \\
\hline No & $45 / 60(75.0)$ & $1.59 \pm 0.20$ & 0.42 & $22 / 29(75.9)$ & $-0.08 \pm 0.09$ & 0.80 \\
\hline \multicolumn{7}{|c|}{$\mathrm{SSBs}^{\mathrm{a}} \geq 3 \times /$ week at 2 years } \\
\hline Yes & $7 / 59(11.9)$ & $1.47 \pm 0.23$ & & $4 / 30(13.3)$ & $-0.12 \pm 0.05$ & \\
\hline No & $52 / 59(88.1)$ & $1.59 \pm 0.19$ & 0.13 & $26 / 30(86.7)$ & $-0.07 \pm 0.10$ & 0.81 \\
\hline \multicolumn{7}{|c|}{ Flavored milks at 12 months } \\
\hline Yes & $10 / 60(16.7)$ & $1.53 \pm 0.29$ & & $7 / 29(24.1)$ & $-0.07 \pm 0.12$ & \\
\hline No & $50 / 60(83.3)$ & $1.59 \pm 0.20$ & 0.38 & $22 / 29(75.9)$ & $-0.08 \pm 0.09$ & 0.36 \\
\hline \multicolumn{7}{|c|}{ Flavored milks $\geq 3 \times /$ week at 24 months } \\
\hline Yes & $13 / 59(22.0)$ & $1.62 \pm 0.21$ & 0.36 & $9 / 30(30.0)$ & $-0.10 \pm 0.10$ & \\
\hline No & $46 / 59(78.0)$ & $1.57 \pm 0.20$ & & $21 / 30(70.0)$ & $-0.08 \pm 0.09$ & 0.95 \\
\hline
\end{tabular}

aSSB (sugar sweetened beverages) consumption defined as soda, Kool-Aid, Hi_C, sweetened juices, and other beverages with added sugar.

SSB, sugar sweetened beverages.

ethnicity, advanced maternal age, maternal age (continuous), or marital status (Table 1). Females had slightly longer telomeres, but the results were not statistically significant $(1.62 \pm 0.18$ vs. $1.55 \pm .21 ; p=0.20)$. There was no association between child's age and telomere length measurement at 2 years of age (Beta Coeff $=-0.03,95 \%$ CI $[-0.06$ to $0.06] ; p=0.59$ ). Measurement at 2 years of age was highly correlated with measurement at birth (Beta Coeff $=0.54$, 95\% CI [0.27 to 0.81$] ; p<0.01)$.

Obesity. Children who were obese at 6 months of age had shorter telomere than those who were not obese, with marginal statistical significance $(1.60 \pm 0.20$ vs. $1.46 \pm 0.14$; $p=0.06$ ) (Table 2). We found no significant differences in telomere length in relation to obesity or waist circumference at other time points in infancy and early childhood and no differences based on breastfeeding duration, exclusivity, or timing or frequency of consumption of beverages. We also did not find any association between birthweight in grams $(p=0.80)$ or birth weight for length $\mathrm{Z}$ score in relation to telomere length at age $2-3(p=0.95)$.

Sugar-sweetened beverages. By 2 years of age, $61.5 \%$ were drinking sodas and SSB in contrast with 96.3\% who were drinking $100 \%$ fruit juice. Mean frequency of consumption of SSBs was low at $4.69 \pm 6.73$ times per

\section{Table 3. Multivariable Predictors of Leukocyte Telomere Length (T/S Ratio)}

at 2-3 Years of Age $(n=58)$

\begin{tabular}{|c|c|c|c|}
\hline Variable & $\beta$-Coefficient & 95\% Confidence interval & $p$ \\
\hline Obese at $2-3$ years & -0.59 & -0.22 to 0.10 & 0.46 \\
\hline Obese at 6 months & -0.14 & -0.29 to 0.11 & 0.07 \\
\hline Male sex & -0.06 to 0.50 & -0.16 to 0.04 & 0.21 \\
\hline SSB, freq of consumption at 2 years ${ }^{a}$ & -0.009 & -0.02 to -0.0008 & 0.03 \\
\hline Age at telomere collection & -0.05 & -0.14 to 0.03 & 0.24 \\
\hline
\end{tabular}

All listed variables are adjusted for in the above model.

Bold denotes statistical significance.

${ }^{a}$ Total number of times that child consumes SSBs over a I-month period measured continuously. 


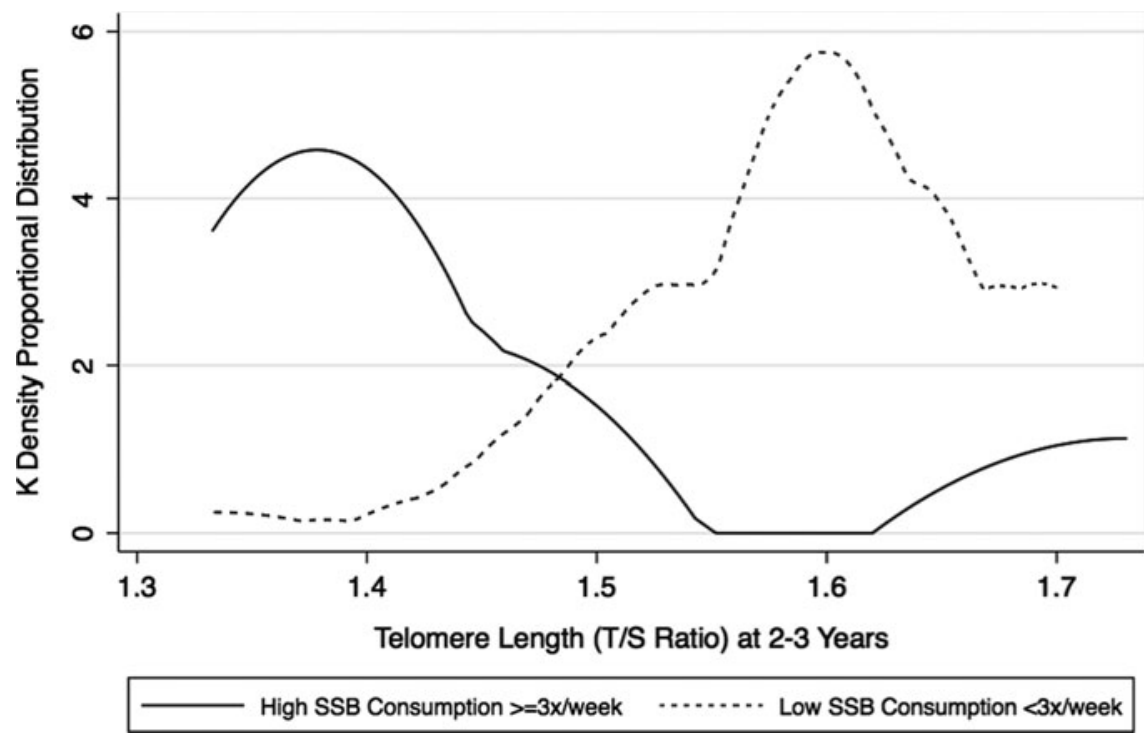

Figure 1. Proportional distribution of telomere length by SSB consumption in Latino preschool children. SSB, sugar-sweetened beverages.

month in contrast with $100 \%$ fruit juice at $13.94 \pm-9.81$ times per month. SSB consumption ( $\geq 3$ times per week at 2 years of age) was associated with reduced telomere length, but results were not statistically significant (1.59 \pm 0.19 vs. $1.47 \pm 0.23 ; p=0.13)$ (Table 2$)$.

\section{Telomere length attrition from 0 to 2 years}

Mean yearly change in telomere length was $-0.08 \pm 0.09$ $\mathrm{T} / \mathrm{S}$ ratio from 0 to $2-3$ years of age. We had only 30 children that had repeat samples from 0 to 2-3 years of age to measure change. We did not see any difference between rate of attrition and child sex, maternal ethnicity, age, or marital status (Table 1). Children of mothers who had a high-school diploma appeared to have faster rates of attrition compared with those who did not $(-0.06 \pm 0.09$ vs. $-0.15 \pm 0.09)$, although results were not statistically significant $(p=0.06)$.

We did not find any significant differences in rate of telomere attrition based on childhood weight status at different points in childhood (Table 2) or beverage intake. Children who were introduced $100 \%$ fruit juice by 6 months of age appeared to have more accelerated shortening, but the results were not statistically significant $(-0.13 \pm 0.06$ vs. $-0.05 \pm 0.17 ; p=0.10$ ) (Table 2).

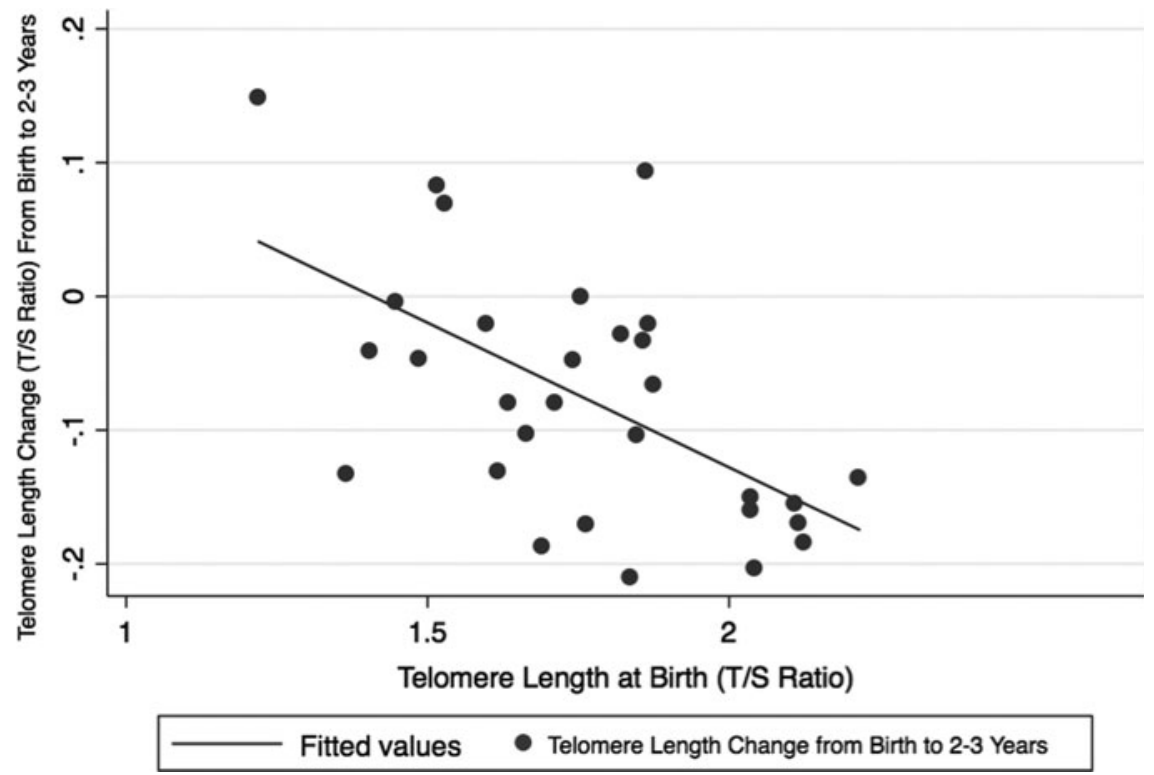

Figure 2. Telomere length change in relation to telomere length at birth. 


\section{Multivariable Regression}

We use multivariable regression models to assess independent predictors for telomere length at age 2-3, including obesity at 6 months and 2-3 years of age, SSB consumption at 2 years, gender, and age of telomere collection at 2-3 years into our model. This model showed that consuming higher levels of SSB was significantly associated with reduced telomere length (Beta Coeff $=-0.009$, 95\% [CI -41.80 to -0.02 to -0.0008$] ; p=0.03$ ) (Table 3 and Fig. 1 ).

In a separate model, we included an interaction term between obesity and increasing SSB consumption that was significant (Beta Coeff for interaction term between obesity and $\mathrm{SSB}=0.04,0.006-0.07 ; p=0.02$ ), indicating that the effect of higher SSB consumption was greater among nonobese children (Beta Coeff $=-0.012,95 \%$ [CI -0.02 to $-0.004] ; p<0.01)$.

We conducted a second multivariable regression model so as to investigate risk factors for accelerated attrition, finding that telomere length at birth strongly predicted telomere length at 2-3 years of age with increasing length at birth associated with accelerated attrition (Beta Coeff $=$ $-0.21,95 \%$ CI -0.34 to -0.07 ) (Fig. 2; Table 4). Male sex bordered on statistical significance for having accelerated attrition (Beta Coeff $=-0.06,95 \%$ CI $-0.12-0.004$ ).

\section{Discussion}

\section{Sugar-Sweetened Beverages}

This is the first study to suggest that increasing amounts of SSB can have a deleterious impact on cellular health by age $2-3$ as indicated by shorter LTL. The relationship was not significant when examined without adjusting for confounding factors such as age at collection, sex, and BMI. However, in the multivariate model, after adjusting for these factors, SSB intake was significantly related to shorter TL. In addition, we found that this relationship was stronger in the nonobese children. Nonobese children who drank more sugary drinks were more likely to have shorter telomeres.

While adult studies have similarly found an association between excessive SSB consumption and shorter LTL, ${ }^{10}$ to find an association at such a young age, particularly in children who are not obese, stresses the importance of preventing and delaying consumption of SSBs in preschool children. Furthermore, as the mean monthly consumption on average in our cohort was relatively low $(4.7 \pm 6.7$ servings per month), and those that consumed SSB three or more times per week was also low at $11.9 \%$, our findings indicate that even a minimal amount of SSBs can have a negative impact. Even at this low rate, SSBs adversely impacted cellular health, particularly in children who were not yet obese.

\section{Differential Impact of SSB on Nonobese Children}

Previous studies with preschool age children have found that SSB consumption is only associated with obesity as children reach older preschool age (4 and 5 years vs. 2-3 years). ${ }^{4}$ Furthermore, increased SSB intake may first impact cardiometabolic indicators of health, including cholesterol levels, fasting insulin and glucose, and waist circumference before impacting BMI in older children..$^{20,21}$

Meanwhile, we did not see any association between telomere length and waist circumference at 2-3 years of age, waist circumference $>90$ th percentile, or obesity at 2-3 years of age. Early obesity at 6 months of age neared statistical significance for association with shorter telomere length at age 2-3 similar to our previous study with older children in the Hispanic, Eating and Nutrition (HEN) cohort. ${ }^{9}$ Telomere length may be particularly sensitive to inflammation and reactive oxygen species damage in infancy and early childhood, and these processes may happen before the onset of obesity. It is not clear why SSB consumption had a stronger effect on the leukocyte telomeres of those children who were not yet obese at 2-3 years. We did not adjust for total energy intake in the children, as it was not available for the majority of the sample, so it is possible that obese children may have had higher overall energy intake, eclipsing the overall significance of SSB, in contrast with nonobese children

Meanwhile, however, the differential impact of SSB consumption on telomere length in obese versus nonobese children indicates the complexity of metabolic disease development. Increased cytokine production from larger adipocytes may be responsible for a higher inflammatory burden that may minimize the effect of any SSB consumption, particularly in smaller amounts. ${ }^{22}$ However, children who are not yet obese, may be more sensitive to the

\begin{tabular}{|c|c|c|c|}
\hline Variable & $\beta$-Coefficient & 95\% Confidence interval & $p$ \\
\hline Male sex & -0.06 & -0.12 to 0.004 & 0.06 \\
\hline $100 \%$ fruit juice consumption at 6 months of age & -0.05 & -0.12 to 0.02 & 0.13 \\
\hline$\geq$ High school diploma completed for mother & -0.02 & -0.09 to 0.04 & 0.45 \\
\hline Telomere length at birth, $T / S$ ratio & -0.21 & -0.34 to -0.07 & $<0.01$ \\
\hline
\end{tabular}

All listed variables are adjusted for in the above model. 
deleterious impact of SSB, as they do not have the same high inflammatory burden as obese children. We did not find any difference in SSB consumption patterns between obese and nonobese children at 2 years of age, with both having relatively low rates of consumption $(3 \pm 4.5$ vs. $4.6 \pm 6.5$ servings per month; $p=0.45$ ).

\section{Telomere Attrition from Birth to 2 Years of Age}

This is also the first study to evaluate attrition from birth to 2 years of age finding that the most significant predictor of attrition rate in early childhood is telomere length at birth. This finding may be related to the phenomenon of regression to the mean. Another study of cord blood telomere length at birth found that the variance at birth in telomere length was similar to that for adults, suggesting that much of our telomere length profile is created in utero, ${ }^{23}$ and stresses the importance of better understanding those factors that impact telomere length shortening in utero. Male children also had greater attrition than females in the first 2-3 years of life, although the results bordered on statistical significance $(p=0.06)$. Female children had slightly longer telomeres at birth as we and others have shown, ${ }^{23,14}$ although ours is the first to suggest that in addition to shorter telomeres at birth, males may have accelerated attrition. Notably this is in spite of the well-known effect that longer baseline telomere length leads to greater attrition.

\section{Limitations}

Our results are preliminary as our sample size was small and further studies need to be conducted in larger population groups. Furthermore, while Latinos are at particularly high risk for obesity, particularly in comparison with nonHispanic whites, additional studies are needed with diverse, multiethnic groups so as to compare the differential impact of environmental exposures such as SSB on early risk for obesity and metabolic disease. Additional studies are also needed that adjust for total energy intake in addition to SSB intake. Follow-up studies, to assess weight gain and metabolic disease development, are needed, as our group is currently conducting, to better understand the stronger association we found with nonobese children for SSB consumption and shorter telomere length. Lastly, we used quantitative polymerase chain reaction-based methods to assess telomere length, which some studies have found to have a larger measurement error than Southern blots. ${ }^{24,} 25$ Additional studies are needed using other methods to assess telomere length including Southern blot.

\section{Acknowledgments}

Allen Foundation, NIH NIDDK 097458, Marc and Lynne Benioff, and Hellman Family Foundation supported this work.

\section{Author Disclosure Statement}

JL is a consultant to Telomere Diagnostics, Inc., formerly Telomere Health, and owns stock in the company, and the company did not play any role in this research. The remaining authors declare no conflicts of interest.

\section{References}

1. Scharf RJ, DeBoer MD. Sugar-sweetened beverages and children's health. Annu Rev Public Health 2016;37:273-293.

2. Keller A, Bucher Della Torre S. Sugar-sweetened beverages and obesity among children and adolescents: A review of systematic literature reviews. Child Obes 2015;11:338-346.

3. Cantoral A, Tellez-Rojo MM, Ettinger AS, et al. Early introduction and cumulative consumption of sugar-sweetened beverages during the pre-school period and risk of obesity at 8-14 years of age. Pediatr Obes 2016;11:68-74.

4. DeBoer MD, Scharf RJ, Demmer RT. Sugar-sweetened beverages and weight gain in 2-to-5 year old children. Pediatrics 2013;132:415.

5. D’Mello MJ, Ross SA, Briel M, et al. Association between shortened leukoycte telomere length and cardiometabolic outcomes: Systematic review and meta-analysis. Circ Cardivoasc Genet 2015;8:82-90.

6. Leung CW, Lariaia BA, Coleman-Phox K, et al. Sugary beverage and food consumption, and leukocyte telomere length maintenance in pregnant women. Eur J Clin Nutr 2016;70:1086-1088.

7. Nettleton JA, Diez-Rouz A, Jenny NS, et al. Dietary patterns, food groups, and telomere length in the Multi-Ethnic Study of Atherosclerosis (MES). Am J Clin Nutr 2008;88:1405-1412.

8. Lee JY, Jun NR, Yoon D, et al. Association between dietary patterns in the remote past and telomere length. Eur J Clin Nutr 2015; 69:1048-1052.

9. Wojcicki JM, Heyman MB, Elwan D, et al. Early exclusive breastfeeding is associated with longer telomeres in Latino preschool children. Am J Clin Nutr 2016;104:397-405.

10. Leung CW, Laraia BA, Needham BL, et al. Soda and cell aging: Associations between sugar-sweetened beverage consumption and leukocyte telomere length in healthy adults from the National Health and Nutrition Examination Surveys. Am J Public Health 2014; 104:2425-2431.

11. Wojcicki JM, Shiboski S, Heyman MB, et al. Telomere length change plateaus at 4 years of age in Latino children: Associations with baseline telomere length and maternal change. Mol Genet Genomics 2016;291:137-189.

12. Frenck RW, Blackburn EH, Shannon KM. The rate of telomere sequence loss in human leukocytes varies with age. Proc Natl Acad Sci U S A 1998;95:5607-5610.

13. Rufer N, Brummendorf TH, Kolvraa S, et al. Telomere fluorescence measurements in granulocytes and $\mathrm{T}$ lymphocyte subsets point to high turnover of stem cells and memory $\mathrm{T}$ cells in early childhood. J Exp Med 1999;190:157-167.

14. Wojcicki JM, Olveda R, Heyman MB, et al. Cord blood telomere length in Latino infants: Relation with maternal education and infant sex. J Perinatol 2016;36:235-241.

15. Ville AP, Heyman MB, Medrano R, Wojcicki JM. Early antibiotic exposure and risk of childhood obesity in Latinos. Child Obes 2017;13:231-235.

16. Cawthon RM. Telomere length measurement by quantitative PCR. Nucl Acids Res 2002;30:e47.

17. Lin J, Epel E, Cheon J, et al. Analyses and comparisons of telomerase activity and telomere length in human T and B cells: Insights from epidemiology of telomere maintenance. J Immunol Methods 2010;3521:71-80. 
18. Kuczmarski RJ, Ogden CL, Guo SS, et al. 2000 CDC growth charts for the United States: Methods and development. National Center for Health Statistics. Vital Health Stat 11 2002;246:1-190.

19. Fryar CD, Gu Q, Ogden CL. Anthropometric reference data for children and adults: United States, 2007-2010. National Center for Health Statistics. Vital Health Stats 11 2012;252:1-48.

20. Kosova EC, Auinger P, Bremer AA. The Relationships between sugar-sweetened beverage intake and cardiometabolic markers in young children. J Acad Nutr Diet 2013;113:219-227.

21. Lin WT, Chan TF, Huang HL, et al. Fructose-rich beverage intake and central adiposity, uric acid and pediatric insulin resistance. $J$ Pediatr 2016;171:90-96.

22. Greenberg AS, Obin MS. Obesity and the role of adipose tissue in inflammation and metabolism. Am J CLin Nutr 2006;83:46154655.

23. Factor-Litvak P, Susser E, Kezio K, et al. Leukocyte telomere length in newborns: Implications for the role of telomeres in human disease. Pediatrics 2016;137:e20153927.
24. Elbers CC, Garcia ME, Kimura M, et al. Comparison between southern blots and qPCR analysis of leukocyte telomere length in the health ABC study. J Gerontol A BiolSci Med Sci 2014;69:527531.

25. Aviv A, Hunt SC, Lin J, et al. Impartial comparative analysis of measurement of leukocyte telomere length/DNA content by southern blots and qPCR. Nucleic Acids Res 2011;39: e134.

Address correspondence to:

Janet M. Wojcicki, PhD, MPH, MAS

Department of Pediatrics

University of California, San Francisco 550 16th Street, 5th Floor San Francisco, CA 94134-0136

E-mail: Wojcicki@gmail.com 